\title{
Test of Lorentz Invariance in Electrodynamics Using Rotating Cryogenic Sapphire Microwave Oscillators
}

\author{
Paul L. Stanwix ${ }^{1}$ * Michael E. Tobar ${ }^{1}$ 円 Peter Wolf ${ }^{2,3}$, Mohamad Susli ${ }^{1}$, Clayton \\ R. Locke ${ }^{1}$, Eugene N. Ivanov ${ }^{1}$, John Winterflood ${ }^{1}$, and Frank van Kann ${ }^{1}$ \\ ${ }^{1}$ University of Western Australia, School of Physics M013, 35 Stirling Hwy., Crawley 6009 WA, Australia \\ ${ }^{2}$ SYRTE, Observatoire de Paris, 61 Av. de l'Observatoire, 75014 Paris, France \\ ${ }^{3}$ Bureau International des Poids et Mesures, Pavillon de Breteuil, 92312 Sèvres Cedex, France
}

(Dated: July 20, 2021)

\begin{abstract}
We present the first results from a rotating Michelson-Morley experiment that uses two orthogonally orientated cryogenic sapphire resonator-oscillators operating in whispering gallery modes near $10 \mathrm{GHz}$. The experiment is used to test for violations of Lorentz Invariance in the frame-work of the photon sector of the Standard Model Extension (SME), as well as the isotropy term of the Robertson-Mansouri-Sexl (RMS) framework. In the SME we set a new bound on the previously unmeasured $\tilde{\kappa}_{e-}^{Z Z}$ component of $2.1(5.7) \times 10^{-14}$, and set more stringent bounds by up to a factor of 7 on seven other components. In the RMS a more stringent bound of $-0.9(2.0) \times 10^{-10}$ on the isotropy parameter, $P_{M M}=\delta-\beta+\frac{1}{2}$ is set, which is more than a factor of 7 improvement.

PACS numbers: 03.30.+p, 06.30.Ft, 12.60.-i, 11.30.Cp, 84.40.-x
\end{abstract}

The Einstein Equivalence Principle (EEP) is a founding principle of relativity [1]. One of the constituent elements of EEP is Local Lorentz Invariance (LLI), which postulates that the outcome of a local experiment is independent of the velocity and orientation of the apparatus. The central importance of this postulate has motivated tremendous work to experimentally test LLI. Also, a number of unification theories suggest a violation of LLI at some level. However, to test for violations it is necessary to have an alternative theory to allow interpretation of experiments [1] and many have been developed 2, 3, 4, 5, 6, 7]. The kinematical Roberson-MansouriSexl (RMS) 2, 3] framework postulates a simple parameterization of the Lorentz transformations with experiments setting limits on the deviation of those parameters from their values in special relativity (SR). Because of their simplicity they have been widely used to interpret many experiments 8, 9, 10, 11]. More recently, a general Lorentz violating extension of the standard model of particle physics (SME) has been developed [6] whose Lagrangian includes all parameterized Lorentz violating terms that can be formed from known fields.

This work presents first results of a rotating lab experiment using cryogenic microwave oscillators. Previous non-rotating experiments [10, 12, 13] relied on the earth's rotation to modulate a Lorentz violating effect. This is not optimal for two reasons. Firstly, the sensitivity is proportional to the noise of the oscillators at the modulation frequency, typically best for periods between 10 and 100 seconds. Secondly, the sensitivity is proportional to the square root of the number of periods of the modulation signal, therefore taking a relatively long time to acquire sufficient data. Thus, by rotating the experiment the data integration rate is increased and the relevant signals are translated to the optimal operating regime [14].
Our experiment consists of two cylindrical sapphire resonators of $3 \mathrm{~cm}$ diameter and height supported by spindles within superconducting niobium cavities [15], and are oriented with their cylindrical axes orthogonal to each other in the horizontal plane. Whispering gallery modes [16] are excited near $10 \mathrm{GHz}$, with a difference frequency of $226 \mathrm{kHz}$. The frequencies are stabilized using Pound locking, and amplitude variations are suppressed using an additional control circuit. A detailed description of such oscillators can be found in [17, 18]. The resonators are mounted in a common copper block, which provides common mode rejection of temperature fluctuations. The structure is in turn mounted inside two successive stainless steel vacuum cylinders from a copper post, which provides the thermal connection between the cavities and the liquid helium bath. A foil heater and carbon-glass temperature sensor attached to the copper post controls the temperature set point to $6 \mathrm{~K}$ with $\mathrm{mK}$ stability.

A schematic of the rotation system is shown in Fig 1 The cryogenic dewar along with the room temperature oscillator and control electronics is suspended within a ring bearing. A multiple "V" shaped suspension made from elastic cord avoids high Q-factor pendulum modes by ensuring that the cord has to stretch and shrink (providing damping) for horizontal and vertical motion. The rotation system is driven by a microprocessor controlled stepper motor. A commercial 18 conductor slip ring connector, with a hollow through bore, transfers power and various signals to and from the rotating experiment. A mercury based rotating coaxial connector transmits the difference frequency to a stationary frequency counter referenced to an Oscilloquartz oscillator. The data acquisition system logs the difference frequency as a function of orientation, as well as monitoring systematic effects including the temperature of the resonators, liquid helium 


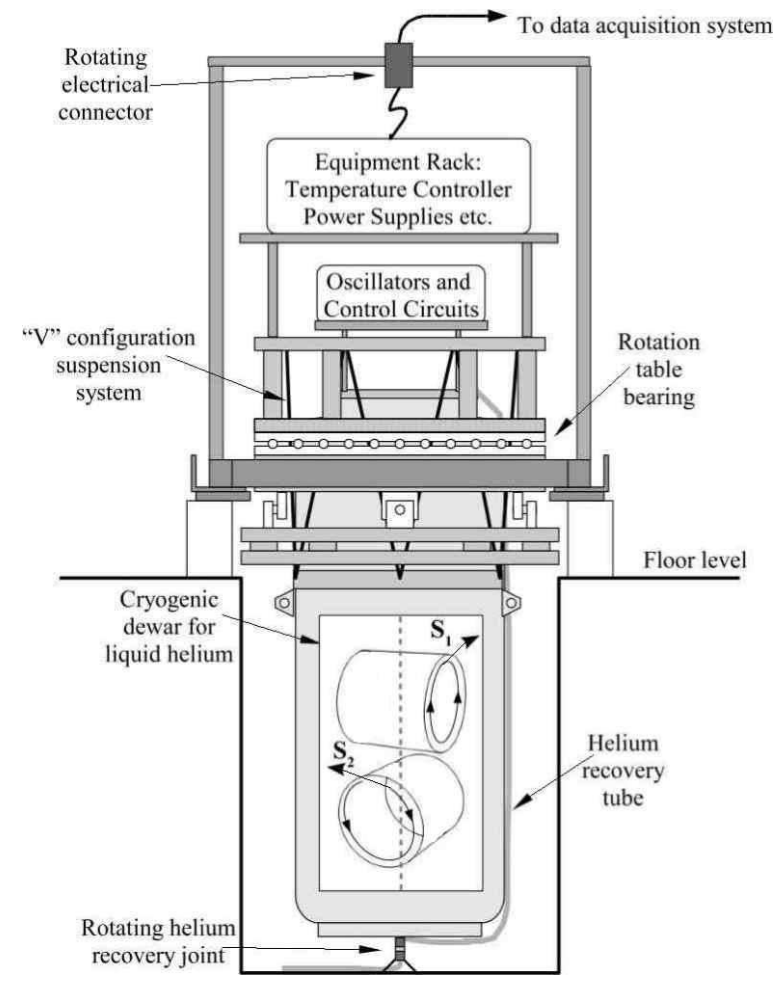

FIG. 1: Schematic of the cryogenic dewar, mounted in the rotation table. Inside the dewar a schematic of the two orthogonally orientated resonators is shown, along with the Poynting vectors of propagation $S_{1}$ and $S_{2}$.

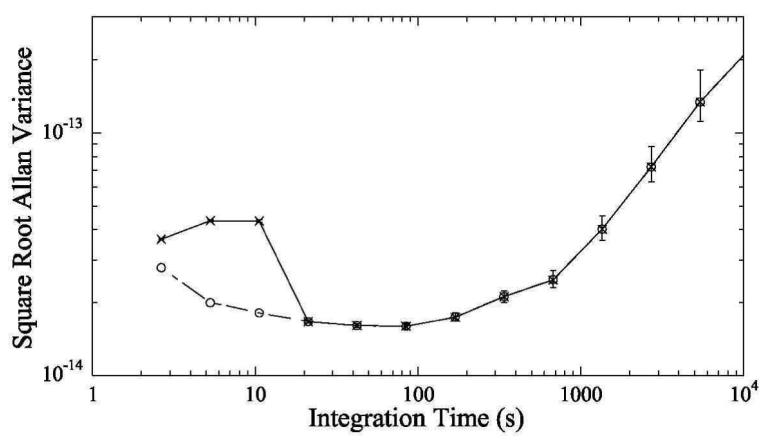

FIG. 2: Square Root Allan Variance fractional frequency instability measurement of the difference frequency when rotating (crosses) and stationary (circles). The hump at short integration times is due to systematic effects associated with the rotation of the experiment, with a period of 18 seconds. Above 18 seconds the instability is the same as when the experiment is stationary.

bath level, ambient room temperature, oscillator control signals, tilt, and helium return line pressure.

Inside the sapphire crystals standing waves are set up with the dominant electric and magnetic fields in the axial and radial directions respectively, corresponding to a Poynting vector around the circumference. The experi- mental observable is the difference frequency, and to test for Lorentz violations the perturbation of the observable with respect to an alternative test theory must be derived. For example, in the photon sector of the SME this may be calculated to first order as the integral over the non-perturbed fields (Eq. (34) of 7]), and expressed in terms of 19 independent variables (discussed in more detail later). The change in orientation of the fields due to the lab rotation and Earth's orbital and sidereal motion induces a time varying modulation of the difference frequency, which is searched for in the experiment. Alternatively, with respect to the RMS framework, we analyze the change in resonator frequency as a function of the Poynting vector direction with respect to the velocity of the lab through the cosmic microwave background (CMB). The RMS parameterizes a possible Lorentz violation by a deviation of the parameters $(\alpha, \beta, \delta)$ from their SR values $\left(-\frac{1}{2}, \frac{1}{2}, 0\right)$. Thus, a complete verification of LLI in the RMS framework 2, 3] requires a test of (i) the isotropy of the speed of light $\left(P_{M M}=\delta-\beta+\frac{1}{2}\right)$, a Michelson-Morley (MM) experiment [19], (ii) the boost dependence of the speed of light $\left(P_{K T}=\beta-\alpha-1\right)$, a Kennedy-Thorndike (KT) experiment 20] and (iii) the time dilation parameter $\left(P_{I S}=\alpha+\frac{1}{2}\right)$, an Ives-Stillwell (IS) experiment 21, 22]. Because our experiment compares two cavities it is only sensitive to $P_{M M}$.

Fig 2 shows typical fractional frequency instability of the $226 \mathrm{kHz}$ difference with respect to $10 \mathrm{GHz}$, and compares the instability when rotating and stationary. A minimum of $1.6 \times 10^{-14}$ is recorded at 40 s. Rotation induced systematic effects degrade the stability up to $18 \mathrm{~s}$ due to signals at the rotation frequency of $0.056 \mathrm{~Hz}$ and its harmonics. We have determined that tilt variations dominate the systematic by measuring the magnitude of the fractional frequency dependence on tilt and the variation in tilt at twice the rotation frequency, $2 \omega_{R}(0.11 \mathrm{~Hz})$, as the experiment rotates. We minimize the effect of tilt by manually setting the rotation bearing until our tilt sensor reads a minimum at $2 \omega_{R}$. The latter data sets were up to an order of magnitude reduced in amplitude as we became more experienced at this process. The remaining systematic signal is due to the residual tilt variations, which could be further annulled with an automatic tilt control system. It is still possible to be sensitive to Lorentz violations in the presence of these systematics by measuring the sidereal, $\omega_{\oplus}$ and semi-sidereal, $2 \omega_{\oplus}$ sidebands about $2 \omega_{R}$, as was done in [8]. The amplitude and phase of a Lorentz violating signal is determined by fitting the parameters of $\mathrm{Eq}$ to the data, with the phase of the fit adjusted according to the test theory used.

$$
\frac{\Delta \nu_{0}}{\nu_{0}}=A+B t+\sum_{i} C_{i} \cos \left(\omega_{i} t+\varphi_{i}\right)+S_{i} \sin \left(\omega_{i} t+\varphi_{i}\right)
$$

Here $\nu_{0}$ is the average unperturbed frequency of the two sapphire resonators, and $\Delta \nu_{0}$ is the perturbation of the $226 \mathrm{kHz}$ difference frequency, $A$ and $B$ determine the 


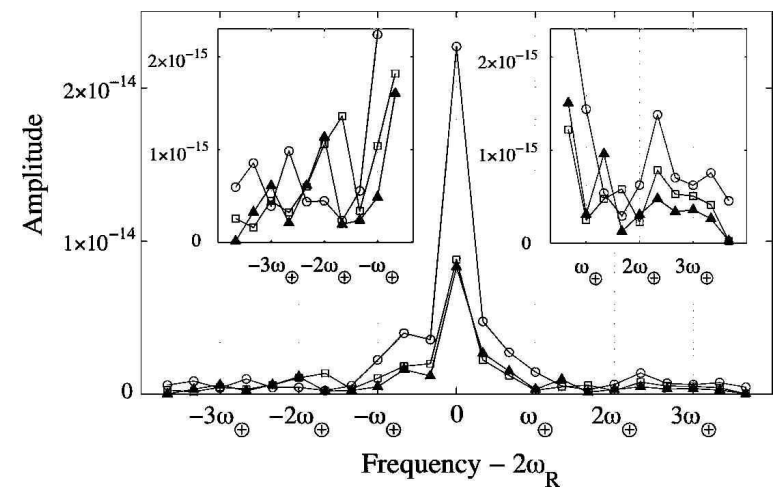

FIG. 3: Spectrum of amplitudes $\sqrt{C_{i}^{2}+S_{i}^{2}}$ calculated using WLS, showing systematic leakage about $2 \omega_{R}$ for 2 data sets, data set 1 (3.6 days, circles), data set 5 (6.1 days, squares) and the combined data (18 days spanning 3 months, solid triangles). Here $\omega_{\oplus}$ is the sidereal frequency $(11.6 \mu \mathrm{Hz})$. By comparing a variety of data sets we have seen that leakage is reduced in longer data sets with lower systematics. The insets show the typical amplitude away from the systematic, which have statistical uncetainties of order $10^{-16}$.

frequency offset and drift, and $C_{i}$ and $S_{i}$ are the amplitudes of a cosine and sine at frequency $\omega_{i}$ respectively. In the final analysis we fit 5 frequencies to the data, $\omega_{i}=\left(2 \omega_{R}, 2 \omega_{R} \pm \omega_{\oplus}, 2 \omega_{R} \pm 2 \omega_{\oplus}\right)$, as well as the frequency offset and drift. The correlation coefficients between the fitted parameters are all between $10^{-2}$ to $10^{-5}$. Since the residuals exhibit a significantly non-white behavior, the optimal regression method is weighted least squares (WLS) [13]. WLS involves pre-multiplying both the experimental data and the model matrix by a whitening matrix determined by the noise type of the residuals of an ordinary least squares analysis.

We have acquired 5 sets of data over a period of 3 months beginning December 2004, totaling 18 days. The length of the sets (in days) and size of the systematic are $\left(3.6,2.3 \times 10^{-14}\right),\left(2.4,2.1 \times 10^{-14}\right),\left(1.9,2.6 \times 10^{-14}\right)$, $\left(4.7,1.4 \times 10^{-15}\right)$, and $\left(6.1,8.8 \times 10^{-15}\right)$ respectively. We have observed leakage of the systematic into the neighboring side bands due to aliasing when the data set is not long enough or the systematic is too large. Fig 3 shows the total amplitude resulting from a WLS fit to 2 of the data sets over a range of frequencies about $2 \omega_{R}$. It is evident that the systematic of data set 1 at $2 \omega_{R}$ is affecting the fitted amplitude of the sidereal sidebands $2 \omega_{R} \pm \omega_{\oplus}$ due to its relatively short length and large systematics. By analyzing all five data sets simultaneously using WLS the effective length of the data is increased, reducing the width of the systematic sufficiently as to not contribute significantly to the sidereal and semi-sidereal sidebands.

In the photon sector of the SME [7], Lorentz violating terms are parameterized by 19 independent components, which are in general grouped into three traceless and symmetric $3 \times 3$ matrices $\left(\tilde{\kappa}_{e+}, \tilde{\kappa}_{o-}\right.$, and $\left.\tilde{\kappa}_{e-}\right)$, one antisymmetric matrix $\left(\tilde{\kappa}_{o+}\right)$ and one additional scalar, which all vanish when LLI is satisfied. To derive the expected signal in the SME we use the method of 7, 11 to calculate the frequency of each resonator in the SME and in the resonator frame. We then transform to the standard celestial frame used in the SME 7] taking into account the rotation in the laboratory frame in a similar way to 23]. The resulting relation between the parameters of the SME and the $C_{i}$ and $S_{i}$ coefficients are given in Tab【 which, for short data sets, were calculated using the leading order expansion at the annual phase position of the data. The 10 independent components of $\tilde{\kappa}_{e+}$ and $\tilde{\kappa}_{o-}$ have been constrained by astronomical measurements to $<2 \times 10^{-32}$ [7, 25]. Seven components of $\tilde{\kappa}_{e-}$ and $\tilde{\kappa}_{o+}$ have been constrained in optical and microwave cavity experiments [10, 13] at the $10^{-15}$ and $10^{-11}$ level respectively, while the scalar $\tilde{\kappa}_{t r}$ component recently had an upper limit set of $<10^{-4}[23]$. The remaining $\tilde{\kappa}_{e-}^{Z Z}$ component could not be previously constrained in nonrotating experiments [10, 13].

In contrast, our rotating experiment is sensitive to $\tilde{\kappa}_{e-}^{Z Z}$. However, it appears only at $2 \omega_{R}$, which is dominated by systematic effects. From our combined analysis of all data sets, and using the relation to $\tilde{\kappa}_{e-}^{Z Z}$ given in Tab【 we determine a value for $\tilde{\kappa}_{e-}^{Z Z}$ of $4.1(0.5) \times 10^{-15}$. However, since we do not know if the systematic has canceled a Lorentz violating signal at $2 \omega_{R}$, we cannot reasonably claim this as an upper limit. Since we have five individual data sets, a limit can be set by treating the $C_{2 \omega_{R}}$ coefficient as a statistic. The phase of the systematic depends on the initial experimental conditions, and is random across the data sets. Thus, we have five values of $C_{2 \omega_{R}},\left(\{-4.2,11.4,21.4,1.3,-8.1\}\right.$ in $\left.10^{-15}\right)$. If we take the mean of these coefficients, the systematic signal will cancel if its phase is random, but the possible Lorentz violating signal (with constant phase) will not. Thus a limit can be set by taking the mean and standard deviation of the five coefficient of $C_{2 \omega_{R}}$. This gives a more conservative bound of 2.1(5.7) $\times 10^{-14}$, which includes zero. Our experiment is also sensitive to all other seven components of $\tilde{\kappa}_{e-}$ and $\tilde{\kappa}_{o+}$ (see Tab【) and improves present limits by up to a factor of 7 , as shown in Tab III

In the RMS frame-work, a frequency shift due to a putative Lorentz violation is given by $\mathrm{Eq} 2,2$, 11],

$$
\frac{\Delta \nu_{0}}{\nu_{0}}=\frac{P_{M M}}{2 \pi c^{2}}\left[\oint\left(\mathbf{v} \cdot \hat{\theta}_{\mathbf{1}}\right)^{2} d \varphi_{1}-\oint\left(\mathbf{v} \cdot \hat{\theta}_{\mathbf{2}}\right)^{2} d \varphi_{2}\right]
$$

Where $\mathbf{v}$ is the velocity of the preferred frame wrt the $\mathrm{CMB}, \hat{\theta}_{j}$ is the unit vector in the direction of the azimuthal angle (direction of propagation) of each resonator (labeled by subscripts 1 and 2), and $\varphi$ is the azimuthal variable of integration in the cylindrical coordinates of each resonator. Perturbations due to Lorentz violations occur at the same five frequencies as the SME, 
TABLE I: Coefficients $C_{i}$ and $S_{i}$ in (1) for the five frequencies of interest and their relation to the components of the SME parameters $\tilde{\kappa}_{e-}$ and $\tilde{\kappa}_{o+}$, derived using a short data set approximation including terms up to first order in orbital velocity, where $\Phi_{0}$ is the phase of the orbit since the vernal equinox (see [24] for details of the calculation). Note that for short data sets the upper and lower sidereal sidebands are redundant, which reduces the number of independent measurements to 5 . To lift the redundancy, more than a year of data is required so annual offsets may be de-correlated from the twice rotational and sidereal sidebands listed.

\begin{tabular}{ccc}
\hline \hline$\omega_{i}$ & $C_{i}$ & $S_{i}$ \\
\hline $2 \omega_{R}$ & $0.21 \tilde{\kappa}_{e-}^{Z Z}$ & - \\
$2 \omega_{R}+\omega_{\oplus}$ & $2.5 \times 10^{-5} \sin \Phi_{0} \tilde{\kappa}_{o+}^{X Y}-1.0 \times 10^{-5} \cos \Phi_{0} \tilde{\kappa}_{o+}^{Y Z}$ & $-\cos \Phi_{0}\left[2.3 \times 10^{-5} \tilde{\kappa}_{o+}^{X Y}-1.0 \times 10^{-5} \tilde{\kappa}_{o+}^{X Z}\right]$ \\
& $-0.27 \tilde{\kappa}_{e-}^{X Z}$ & $-0.27 \tilde{\kappa}_{e-}^{Y Z}$ \\
$2 \omega_{R}+2 \omega_{\oplus}$ & $-2.1 \times 10^{-5} \cos \Phi_{0} \tilde{\kappa}_{o Z}^{X+}$ & $-2.3 \times 10^{-5} \sin \Phi_{0} \tilde{\kappa}_{o+}^{X Z}$ \\
& $+2.3 \times 10^{-5} \sin \Phi_{0} \tilde{\kappa}_{o+}^{Y Z}-0.11\left(\tilde{\kappa}_{e-}^{X X}-\tilde{\kappa}_{e-}^{Y Y}\right)$ & $-2.1 \times 10^{-5} \cos \Phi_{0} \tilde{\kappa}_{o+}^{Y Z}-0.23 \tilde{\kappa}_{e-}^{X Y}$ \\
$2 \omega_{R}-\omega_{\oplus}$ & $-0.31 C_{2 \omega_{R}+\omega_{\oplus}}$ & $0.31 S_{2 \omega_{R}+\omega_{\oplus}}$ \\
$2 \omega_{R}-2 \omega_{\oplus}$ & $9.4 \times 10^{-2} C_{2 \omega_{R}+2 \omega_{\oplus}}$ & $-9.4 \times 10^{-2} S_{2 \omega_{R}+2 \omega_{\oplus}}$ \\
\hline \hline
\end{tabular}

TABLE II: Results for the SME Lorentz violation parameters, assuming no cancelation between the isotropy terms $\tilde{\kappa}_{e-}$ (in $\left.10^{-15}\right)$ and first order boost terms $\tilde{\kappa}_{o+}\left(\right.$ in $\left.10^{-11}\right)$ [12].

\begin{tabular}{lcccc}
\hline \hline & $\tilde{\kappa}_{e-}^{X Y}$ & $\tilde{\kappa}_{e-}^{X Z}$ & $\tilde{\kappa}_{e-}^{Y Z}$ & $\left(\tilde{\kappa}_{e-}^{X X}-\tilde{\kappa}_{e-}^{Y Y}\right)$ \\
\hline this work & $-0.63(0.43)$ & $0.19(0.37)$ & $-0.45(0.37)$ & $-1.3(0.9)$ \\
from [13] & $-5.7(2.3)$ & $-3.2(1.3)$ & $-0.5(1.3)$ & $-3.2(4.6)$ \\
\hline \hline & $\tilde{\kappa}_{e-}^{Z Z}$ & $\tilde{\kappa}_{o+}^{X Y}$ & $\tilde{\kappa}_{o+}^{X Z}$ & $\tilde{\kappa}_{o+}^{Y Z}$ \\
\hline this work & $21(57)$ & $0.20(0.21)$ & $-0.91(0.46)$ & $0.44(0.46)$ \\
from [13] & - & $-1.8(1.5)$ & $-1.4(2.3)$ & $2.7(2.2)$ \\
\hline \hline
\end{tabular}

TABLE III: Dominant coefficients in the RMS, using a short data set approximation calculated from Eq2 The measured values of $P_{M M}$ (in $10^{-10}$ ) are shown together with the statistical uncertainties in the bracket. From this data the measured and statistical uncertainty of $P_{M M}$ is determined to be $-0.9(2.0) \times 10^{-10}$, which represents more than a factor of 7 improvement over previous results $2.2(1.5) \times 10^{-9}[10]$.

\begin{tabular}{ccc}
\hline \hline$\omega_{i}$ & $C u_{i}$ & $P_{M M}$ \\
\hline $2 \omega_{R}+\omega_{\oplus}$ & {$\left[\begin{array}{c}-1.13 \times 10^{-7}-3.01 \times 10^{-8} \cos \Phi_{0} \\
\left.+8.83 \times 10^{-9} \sin \Phi_{0}\right] P_{M M}\end{array}\right.$} & $-2.1(7.2)$ \\
& $+30^{-9} \cos \Phi_{0}$ & $62.4(23.3)$ \\
$2 \omega_{R}-\omega_{\oplus}$ & {$\left[3.51 \times 10^{-8}+9.31 \times 10^{-9} \sin \Phi_{0}\right] P_{M M}$} \\
& $-2.73 \times 10^{-9}$ & \\
$2 \omega_{R}+2 \omega_{\oplus}$ & {$\left[4.56 \times 10^{-7}-1.39 \times 10^{-8} \cos \Phi_{0}\right.$} & $-1.3(2.1)$ \\
& $\left.-7.08 \times 10^{-8} \sin \Phi_{0}\right] P_{M M}$ & \\
$2 \omega_{R}-2 \omega_{\oplus}$ & {$\left[4.37 \times 10^{-8}-1.34 \times 10^{-9} \cos \Phi_{0}\right.$} & $-7.5(22.1)$ \\
& $\left.-6.78 \times 10^{-9} \sin \Phi_{0}\right] P_{M M}$ & \\
\hline \hline
\end{tabular}

but for the RMS analysis we do not consider the $2 \omega_{R}$ frequency due to the large systematic, as we only need to put a limit on one parameter. The dominant coefficients are due to only the cosine terms with respect to the CMB right ascension, $C u_{i}$, which are shown in Tab!II

In conclusion, we set bounds on 7 components of the SME photon sector (TabII) and $P_{M M}$ (Tab!II) of the RMS framework, which are up to a factor of 7 more stringent than those obtained from previous experiments. We have also set an upper limit $\left[2.1(5.7) \times 10^{-14}\right]$ on the previously unmeasured SME component $\tilde{\kappa}_{e-}^{Z Z}$. To further improve these results, tilt and environmental controls will be implemented to reduce systematic effects. To remove the assumption that $\tilde{\kappa}_{o+}$ and $\tilde{\kappa}_{e-}$ do not cancel each other, data integration will continue for more than a year. Note added: Two other concurrent experiments have also set some similar limits [26, 27].

This work was funded by the Australian Research Council.

* Electronic address: pstanwix@physics.uwa.edu.au

† Electronic address: mike@physics.uwa.edu.au

[1] Will C. M., Theory and Experiment in Gravitational Physics, revised edition (Cambridge University Press, Cambridge, 1993).

[2] Robertson H.P., Rev. Mod. Phys. 21, 378 (1949).

[3] Mansouri R., Sexl R.U., Gen. Rel. Grav. 8, 497, (1977).

[4] Lightman A.P., Lee D.L., Phys. Rev. D8, 364, (1973).

[5] Ni W.-T., Phys. Rev. Lett. 38, 301, (1977).

[6] Colladay D., Kostelecký V.A., Phys.Rev.D55, 6760, (1997); Colladay D., Kostelecký V.A., Phys.Rev.D58, 116002, (1998)

[7] Kostelecký V.A.,Mewes M.,Phys.Rev.D66,056005,(2002).

[8] Brillet A., Hall J., Phys. Rev. Lett. 42, 549, (1979).

[9] Wolf P. et al., Phys. Rev. Lett. 90, 060402, (2003).

[10] Müller H. et al., Phys. Rev. Lett. 91, 020401, (2003).

[11] Wolf P., et al., Gen. Rel. and Grav., 36, 2351, (2004).

[12] Lipa J.A. et al. Phys. Rev. Lett. 90, 060403, (2003).

[13] Wolf P, et al., Phys. Rev. D70, 051902(R), (2004).

[14] Tobar M.E. et al., Phys. Lett. A 300, 33, (2002).

[15] Giles A.J. et. al., Physica B 165, 145, (1990).

[16] Tobar M.E., Mann A.G., IEEE Trans. Microw. Theory Tech. 39, 2077, (1991).

[17] Mann A.G., Topics in Appl. Phys. 79, 37, (2001)

[18] Hartnett J.G., Tobar M.E., Topics in Appl. Phys. 79, 67, (2001)

[19] Michelson A.A. and Morley E.W., Am. J. Sci. 34, 333 (1887).

[20] Kennedy R.J. and Thorndike E.M., Phys. Rev. B42, 400 (1932).

[21] Ives H.E. and Stilwell G.R., J. Opt. Soc. Am. 28, 215 (1938). 
[22] Saathoff G., et al., Phys. Rev. Lett 91, 190403 (2003).

[23] Tobar M.E. et. al., Phys.Rev.D71,025004,(2005).

[24] Tobar M.E. et. al., to be published in Springer Lecture Notes in Physics 2005: ArXiv: hep-ph/0506200

[25] Kostelecký V.A., and Mewes M., Phys. Rev. Lett. 87,
251304 (2001).

[26] Antonini, M. Okhapkin, E. Göklü and S. Schiller, Phys. Rev. A 71, 050101R 2005.

[27] Herrmann S. et. al., (to be published). 